\title{
Dictionnaire des usages socio-politiques (1770-1815), fascicule 7, Notions théoriques
}

\section{Patrick Fournier}

\section{(2) OpenEdition \\ 1 Journals}

\section{Édition électronique}

URL : https://journals.openedition.org/ahrf/1555

DOI : 10.4000/ahrf.1555

ISSN : 1952-403X

Éditeur :

Armand Colin, Société des études robespierristes

\section{Édition imprimée}

Date de publication : 1 septembre 2004

Pagination : 208-211

ISSN : 0003-4436

\section{Référence électronique}

Patrick Fournier, « Dictionnaire des usages socio-politiques (1770-1815), fascicule 7, Notions

théoriques », Annales historiques de la Révolution française [En ligne], 337 | juillet-septembre 2004, mis en ligne le 15 février 2006, consulté le 23 avril 2022. URL : http://journals.openedition.org/ahrf/1555 ; DOl : https://doi.org/10.4000/ahrf.1555

Ce document a été généré automatiquement le 23 avril 2022.

Tous droits réservés 


\title{
Dictionnaire des usages socio- politiques (1770-1815), fascicule 7, Notions théoriques
}

\author{
Patrick Fournier
}

\section{RÉFÉRENCE}

Dictionnaire des usages socio-politiques (1770-1815), fascicule 7, Notions théoriques, Paris, Champion, 2003, 207 p., ISBN 2-7453-0805-X, 20 e.

1 Poursuivant son exploration de «l'usage de la langue française pendant la Révolution française ", le septième fascicule du Dictionnaire des usages socio-politiques est consacré à sept notions théoriques. Les présentateurs du volume, Jacques Guilhaumou et MarieFrance Piguet, insistent sur les nouveaux objectifs de cette entreprise originale menée depuis le milieu des années 1980 : distinguer notions-pratiques et notions-théoriques (les premières ayant fait l'objet du précédent fascicule publié en 1999) en montrant l'articulation entre des concepts et des pratiques sociales qui permettent de les justifier ; sortir du politique conçu de manière étroite pour étudier le «discours des sciences sociales en formation"; adopter une gamme de sources (dictionnaires, ouvrages divers, journaux, manuscrits...) et un champ chronologique élargis permettant de comprendre l'évolution des usages de termes et de notions au cours des XVIIIe et XIXe siècles, parfois même sur une plus longue durée. L'une des ambitions majeures est de montrer que les notions théoriques ne constituent pas des concepts définis une fois pour toutes et que leur histoire ne se résume pas à celle de leur surgissement: leur contenu évolue en fonction des discours qui les utilisent dans des contextes sociaux et politiques variés. Si les notions théoriques traduisent une prise de conscience de réalités nouvelles et aident à penser les pratiques sociales, elles sont elles-mêmes des instruments utilisés par les acteurs sociaux pour agir sur la réalité. 
2 Le volume est formé de sept monographies dont quatre étudient des notions qui relèvent du langage de l'économie politique en formation ou s'y intègrent : la «langue de l'économie politique » dans un manuscrit de Sieyès (Jacques Guilhaumou), mais aussi le «travail» (Annie Jacob), la «division du travail» (Christophe Salvat) et la "production» (Marie-France Piguet). L'étude du terme "charlatanisme» (Daniel Teysseire) permet de porter un regard neuf sur les relations qu'entretiennent plusieurs champs linguistiques, le médical, le religieux, le philosophique, le politique et le général. Les articles consacrés à la «grande nation" (Jean-Yves Guiomar) et au «socialisme» (Sonia Branca-Rosoff et Jacques Guilhaumou) ont un contenu politique plus classique, mais traduisent parfaitement l'ambition méthodologique du fascicule.

3 La fortune de l'expression "grande nation» de l'été 1797 à l'automne 1799 s'inscrit dans un contexte politique précis, celui de la campagne victorieuse de Bonaparte en Italie qui donne à la France une puissance et une extension territoriale permettant de la comparer à l'ancienne Gaule. Le terme générique de Grande Nation est même forgé en 1798 pour désigner la France dans ses nouvelles frontières. La notion désigne dans un laps de temps très court au moins deux ambitions politiques majeures: une domination de l'Europe et de la Méditerranée, mais aussi une défense des libertés qu'incarne alors le retour rêvé à la Gaule d'avant la conquête romaine dans le cadre d'une pensée historique encore très dépendante du débat sur les origines de la France. Son utilisation fréquente par les néo-jacobins qui soutiennent Bonaparte montre qu'elle sous-tend un projet politique en opposition avec la ligne directrice du Consulat et qu'elle constitue une arme rhétorique efficace dans le cadre de célébrations publiques dont la portée s'affaiblit dès que la situation internationale redevient plus difficile à l'automne 1798. La naissance de l'expression de "grande nation » dans une conjoncture spécifique n'exclut pas l'inscription de la notion dans une histoire plus longue : celle de la recherche des origines qui traverse tout le XVIIIe siècle, celle de l'historicisme du XIXe siècle qui pourrait être la conséquence de l'attitude précédente tout en rompant avec elle. L'usage rhétorique de la "grande nation" serait donc paradoxalement une étape dans la fondation du principe des nationalités. L'originalité de cette réflexion tient notamment au jeu sur la variation des temporalités, faisant de la courte vie d'une notion un événement qui actualise des idées et des principes plus ou moins anciens en fonction d'une réalité nouvelle. Cela conduit toutefois à s'interroger sur le classement de la " grande nation " parmi les notions théoriques : face aux termes et expressions durables de l'économie politique, son utilisation apparaît souvent comme polémique. Si elle ne figure "pratiquement jamais dans les débats "sérieux » des Conseils", n'est-ce pas parce qu'elle recouvre moins une idée politique qu'un moyen pratique d'exporter un modèle révolutionnaire qui est loin de faire l'unanimité en France comme à l'étranger? La nécessité d'étudier la circulation entre pratiques discursives et émergence de «formes particulières de lexicalisation » (J. Guilhaumou) n'en ressort que plus fortement.

4 Les néologismes constituent un mode d'accès privilégié à cette circulation. Grand créateur de mots destinés à cerner les réalités sociales et à favoriser leur réforme, Sieyès forge "socialisme" dans un manuscrit inédit. La signification spécifique du terme doit être replacée dans le contexte prérévolutionnaire des années 1780 : l'effort de création linguistique rendrait pensable le phénomène révolutionnaire. L'événement linguistique permet de formuler des hypothèses sur le développement du champ sémantique de «société » et sur l'effort de définition d'un art politique distinct de la 
sociabilité conçue comme la capacité de l'homme à vivre en société. Le socialisme de Sieyès n'est pas celui qui ressurgit en 1831, mais il aide à comprendre, sans anachronisme, le projet politique de ce grand réformateur car il participerait aux modes d'élaboration du discours révolutionnaire tels qu'ils ont pu être étudiés notamment par Keith Michael Baker et Roger Chartier.

5 La langue de l'économie politique est un domaine vaste et complexe tant les notions forgées au tournant des XVIIIe et XIXe siècles continuent à animer notre réflexion théorique. Sieyès cherche à lui donner une forme ordonnée selon un mode analytique dans un manuscrit inédit que publie dans ce fascicule J. Guilhaumou. En posant la question de la pertinence des termes utilisés par les économistes, Sieyès contribue moins à faire progresser l'économie politique qu'à la faire entrer dans le débat politique concret.

6 L'histoire des notions de travail et de division du travail facilite la compréhension de certaines mutations sociales et politiques qui se produisent entre les XVIIe et XIXe siècles. La valorisation du travail s'accroît au point, selon A. Jacob, de participer à la déstabilisation de la société d'ordres en associant la citoyenneté au travail. Bien que le lien ne soit pas directement effectué entre les articles d'A. Jacob et de C. Salvat, leur rapprochement permet de suggérer que l'évolution de la vision morale du travail accompagne un effort de conceptualisation analytique qui a permis le développement de l'économie politique. Il serait nécessaire d'approfondir l'étude dynamique de l'articulation entre le travail comme valeur et comme moyen d'accroître la richesse : la notion d'utilité sociale apparaîtrait sans doute comme un des fondements des changements intellectuels de cette époque.

7 Retracer l'histoire de la division du travail de la fin du XVIIe siècle au début du XIXe siècle en mesurant l'influence sur les économistes français de l'expression anglaise division of labour permet de souligner l'importance de la distinction notionnelle dans la formation d'un savoir capable de bouleverser non seulement les représentations mais aussi les pratiques économiques. L'influence d'Adam Smith fut à l'origine d'une révolution épistémologique majeure dans l'économie politique française en facilitant le dépassement et le remplacement de la notion de "séparation des travaux » par celle de «division du travail». Les débats engagés par les économistes sur la pertinence de cette dernière notion contribuèrent à affiner la réflexion sur les relations entre les différents types de production, les échanges et les modalités du travail. La question fondamentale est celle de la place de la division du travail dans les processus économiques de création de valeur : avec Adam Smith, elle devient le fondement d'un enchaînement qui produit de la richesse et de l'échange, ce qui va à l'encontre d'une pensée dominante en France qui fait du surplus agricole le moteur de toute l'activité économique.

8 Les mutations des usages économiques «des éléments lexicaux de la famille morphologique de produire» (M.-F. Piguet) constituent aussi un bon témoignage des spécificités de la pensée économique française à la fin du XVIIIe siècle. L'utilisation fréquente de termes comme "stérile » et «non-productif » par les physiocrates est révélatrice du fondement de leur théorie qui ne peut concevoir de création de richesse que dans les productions de la nature. Toutefois, Quesnay et ses émules contribuent à enrichir le vocabulaire de l'économie politique en forgeant et diffusant les néologismes "productif» et «reproduction »: l'évolution de l'usage et du sens de ces termes accompagne dès lors les progrès de l'économie politique. 
9 La cohérence du volume est rendue sensible par les multiples parcours possibles entre les articles. Dans sa présentation, M.-F. Piguet en propose un différent de celui de ce compte rendu. Plusieurs auteurs insistent sur les phénomènes de colinguisme et sur leurs implications pour l'histoire des idées. Le rôle central de certains théoriciens (Quesnay, Sieyès, Jean-Baptiste Say...) dans la formation du vocabulaire français de l'économie politique et de l'» art politique » est rappelé à plusieurs reprises, de même que la circulation des termes et des notions entre la France et l'Angleterre : cela ne surprend pas, mais invite à mener des études transversales plus systématiques mettant en relation pratiques et rhétorique. À la fois instrument de travail et manifeste méthodologique, ce fascicule du Dictionnaire des usages socio-politiques confirme donc l'intérêt de la linguistique pour cerner avec rigueur les changements de mentalité et l'évolution des idées. 